\title{
AVALIAÇÃO DA ESCÓRIA PARA REDUZIR O CONSUMO DE ENERGIA E A INSTABILIDADE ELÉTRICA DO FEA*
}

\author{
Deisi Vieira ${ }^{1}$ \\ Rodolfo Arnaldo Montecinos de Almeida ${ }^{2}$ \\ Wagner Viana Bielefeldt $t^{3}$ \\ Antonio Cezar Faria Vilela ${ }^{4}$
}

\section{Resumo}

Nas aciarias que operam com forno elétrico a arco (FEA) existe o interesse de garantir uma maior estabilidade ao arco elétrico para almejar uma menor distorção no sistema elétrico, consequentemente, uma redução no consumo de energia elétrica. A prática da escória espumante no refino primário do aço líquido melhora consideravelmente a eficiência da transferência de energia do arco elétrico para a carga e promove um menor consumo do refratário, entre outras vantagens. Além disso, a espumação da escória aumenta a estabilidade do arco elétrico, por reduzir o total de distorção harmônica (THD) entre as fases do FEA. No presente trabalho foram coletadas informações de composição química da escória e parâmetros elétricos de 156 corridas de um FEA, de um padrão de carga específico. Com os dados de composição química pode ser determinado o teor de $\mathrm{FeO}$ e basicidade da escória espumante que propiciaram uma redução do THD e no consumo de energia elétrica do FEA. Calculou-se o índice de espumação das escórias. O aumento do índice de espumação proporcionou a queda do THD. A redução do THD mostrou uma redução da energia elétrica consumida na etapa de refino do FEA.

Palavras-chave: Escória espumante; Estabilidade do arco elétrico; Consumo de energia elétrica do FEA.

\section{EVALUATION OF SLAG TO REDUCE ENERGY CONSUMPTION AND ELECTRICAL INSTABILITY OF EAF}

\section{Abstract}

EAF operations have interest to ensure greater stability to the electric arc to aim less distortion in the electrical system, with a consequent reduction in the consumption of electricity. The practice of foamy slag in the primary refining of the liquid steel enhances considerably the electric arc energy transference efficiency and promotes a lower consumption of refractory, between others advantages. Furthermore, the foaming of the slag increases the stability of the electric arc by reducing the THD (total harmonic distortion) between the phases of EAF. In the present study were collected chemical composition of the slag and electrical parameters of 156 heats from an EAF, from a specific charge pattern. Chemical composition data were important in determining the $\mathrm{FeO}$ content and basicity of the foamy slag that enabled a reduction in THD and the power consumption of the EAF. Were calculated the foaming slag index. A foaming slag ratio increase showed a THD decrease. The reduction of THD, for a certain range of heats, showed a reduction of the electric power consumption.

Keywords: Foaming slag; Electric arc stability; Electric power consumption in EAF.

\footnotetext{
Eng. Metalúrgica, mestranda, Laboratório de Siderurgia (LaSid), PPGE3M, UFRGS, Porto Alegre, $R S$, Brasil.

Eng. Metalúrgico, mestrando; LaSid, PPGE3M, UFRGS, Porto Alegre, RS, Brasil.

Prof., Dr.; LaSid; Depto. de Metalurgia, PPGE3M, UFRGS, Porto Alegre, RS, Brasil.

Prof., Dr.-Ing.; LaSid; Depto. de Metalurgia, PPGE3M, UFRGS, Porto Alegre, RS, Brasil.
} 


\section{INTRODUÇÃO}

A presença de cargas não lineares no sistema elétrico do FEA é acompanhada de harmônicos de tensão, provocando quedas de tensão de alimentação dos eletrodos. A soma dos conteúdos dos harmônicos pode ser representada pelo total de distorção harmônica (THD), que está representado na Figura 1. Nota-se que os picos do gráfico mostram que, na fusão da sucata, existe uma forte intensidade de harmônicas de tensão, já na etapa de refino, a estabilidade aumenta de forma significativa, pois diminui o THD [1].

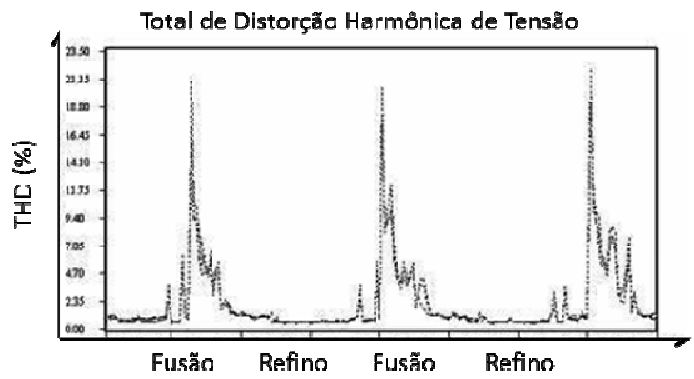

Figura 1. Total de distorção harmônica durante o processo de fusão e refino [1].

A instabilidade gerada pelos harmônicos é maior nos primeiros minutos do processo de fusão. A Figura 2 apresenta a varredura da tensão e da corrente, no início da fusão da sucata, e após a formação da escória espumante. Nota-se uma grande redução dos harmônicos, e um formato de onda mais aproximado da forma senoidal [2].

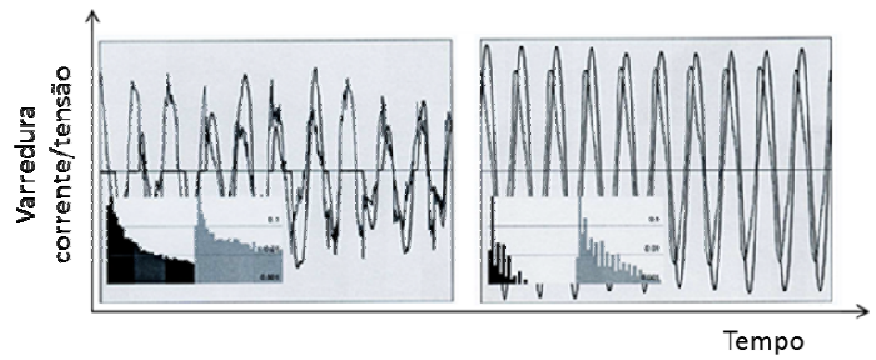

Figura 2. Formas de ondas senoidais, sem a presença de escória espumante (esq.) e com a presença de escória espumante (dir.) [2].

A qualidade da espumação da escória é um fator importante a ser estudado na produção de aço em FEA. A escória espumante contribui com a redução de custos de energia, pois garante que o calor do banho líquido não seja perdido pelas paredes do FEA, mantendo grande parte do calor. Além disso, se for garantida uma boa espumação de escória, a eficiência energética do forno aumenta, há uma diminuição nas vibrações causadas pela fusão, diminuição de som e do consumo de eletrodos. A turbulência gerada durante a fusão resulta em grandes flutuações, não linearidade e instabilidade da corrente do arco elétrico nas diferentes fases. Uma espumação adequada garante que o banho metálico seja coberto, e o caminho feito pela corrente elétrica entre os três eletrodos seja facilitado [3].

Pesquisadores buscam desenvolver modelos úteis para controlar a espumação da escória em processos metalúrgicos e entender a influência da composição química da escória [4]. O índice de espumação ou tempo médio para travessia de um gás na escória é obtido pela razão entre a altura de espuma da escória e a velocidade superficial do gás. Skupien e Gaskell [5] desenvolveram um modelo matemático que 
mostra a forte relação entre o índice de espumação e as propriedades físicas da escória, como a viscosidade, densidade e tensão superficial [5]. O óxido de ferro contido na escória durante a operação do FEA é normalmente formado pela oxidação do ferro quando o oxigênio é injetado no metal fundido. O FeO é um importante componente na escória e tem um efeito significativo sobre viscosidade, capacidade de espumação, volume de escória, produtividade e consumo de energia [7]. Aminorroaya demonstrou em seu estudo que existe uma faixa ótima de FeO para um mínimo consumo de energia elétrica, Figura 3 [8].

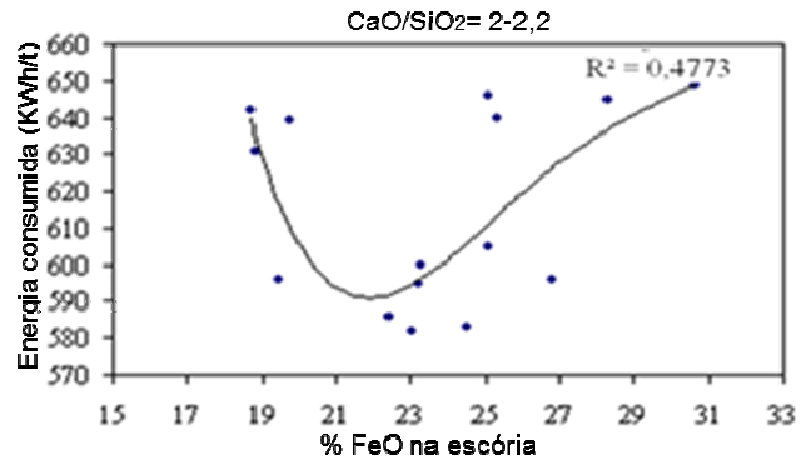

Figura 3. Relação entre o teor de FeO na escória e energia consumida [8].

Já a basicidade binária da escória é um equilíbrio entre os óxidos (principalmente $\mathrm{CaO}$ e $\mathrm{SiO}_{2}$ ). Existem pontos ótimos de basicidade para se alcançar um menor consumo de energia elétrica e a altura máxima de espumação da escória pode estar relacionada com a basicidade, Figura 4 [8].
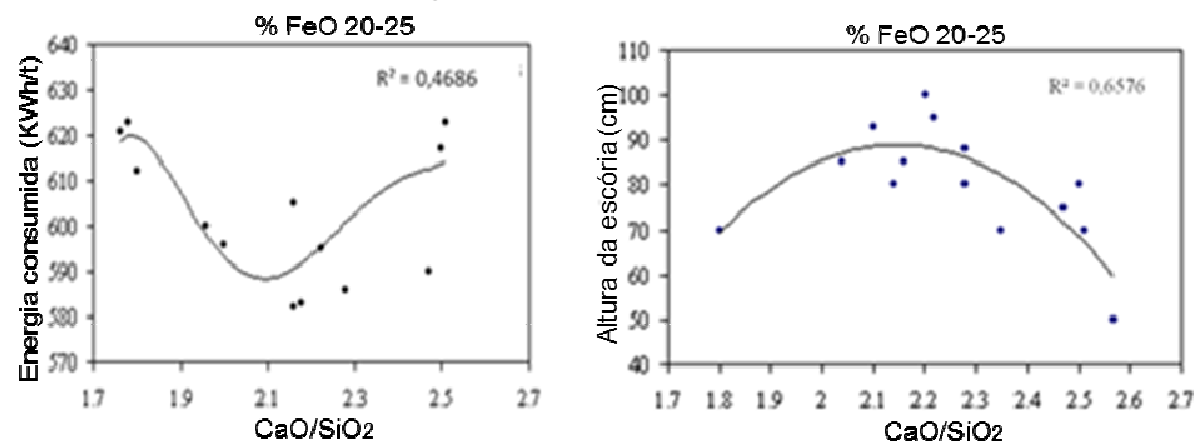

Figura 4. Relação entre basicidade e energia consumida (a) e basicidade e altura da escória (b) [8].

A utilização de uma escória saturada em $\mathrm{MgO}$ é de grande importância nas operações do FEA, formando a escória espumante com maior eficiência, menor custo de energia, maior rendimento do metal e vida útil do refratário mais longa [6]. A quantidade de $\mathrm{MgO}$ na escória provoca um aumento no índice de espumação devido a maior quantidade de partículas sólidas [4].

Este trabalho tem como objetivo relacionar a composição química e o índice de espumação com os parâmetros elétricos de um forno elétrico a arco com vistas a diminuir a instabilidade do arco elétrico e consumo de energia elétrica na etapa de refino.

\section{MATERIAIS E MÉTODOS}

Foram obtidas informações de 156 corridas de aço de um padrão de carga específico, produzidas no período de um ano. O padrão de carga escolhido possui composição química controlada, pois é utilizado para a produção de qualidades 
críticas de aço. Sendo assim, a dispersão das variáveis do processo será minimizada, tanto a composição química quanto a parte de operação, para que os resultados não sejam influenciados pela alta variabilidade dos parâmetros que estão sendo consideradas no presente estudo.

Os dados elétricos analisados foram recebidos diretamente do sistema elétrico de um FEA. Foram feitos histogramas para avaliar a dispersão do "power on" (tempo de forno ligado) e "power off" (tempo de forno desligado) do forno. Foram disponibilizados a tensão e total de distorção harmônica de cada fase (três eletrodos) por segundo durante as corridas, com isso foi calculado o desvio padrão destes dados apenas no intervalo de refino. O consumo de energia elétrica das corridas neste intervalo também foi recebido. A tensão, total de distorção harmônica e o consumo de energia elétrica são fatores que estão relacionados diretamente com a estabilidade do arco elétrico.

A composição química das escórias analisadas foi obtida através da amostra padrão de escória, retirada simultaneamente com a prova de aço em certa etapa durante o refino. Além disso, avaliou-se as faixas dos teores de $\mathrm{FeO}$, basicidade binária e $\mathrm{MgO}$ da escória. Foram comparadas as faixas de composição química do $\mathrm{FeO}$, MgO e a basicidade, onde as corridas tiveram maior concentração, com o consumo de energia elétrica do FEA. As propriedades físicas da escória foram calculadas, pois são essenciais para o cálculo do índice de espumação. A viscosidade foi calculada através do software de simulação termodinâmica FactSage, versão 6.4 [11]. São apresentadas as propriedades, modelos e suas respectivas equações a seguir:

Densidade da escória (Modelo de Mills e Keene) [9] -

$$
\rho=2460+0,18(\% \mathrm{FeO}+\% \mathrm{MnO})(1)
$$

$\rho=$ Densidade da escória;

$\% \mathrm{FeO}=$ percentual em massa de óxido de ferro da escória;

$\% \mathrm{MnO}=$ percentual em massa de óxido de manganês da escória.

Tensão superficial (Modelo de Mills e Keene) [9] -

$\sigma=$ tensão superficial;

$$
\sigma=754,24-569,4\left(\frac{66^{5 i O}}{100}\right)-137,13\left(\frac{96 \mathrm{~F} \theta 0}{100}\right)
$$

$\% \mathrm{SiO}_{2}=$ percentual em massa de óxido sílica presente na escória;

$\% \mathrm{FeO}=$ percentual em massa de óxido de ferro presente na escória.

Viscosidade efetiva (Modelo de Roscoe-Einstein) [10] -

ne - viscosidade efetiva da escória;

$$
\eta_{\mathrm{e}}=\eta(1-\rho \theta)^{-5 / 2}(3)
$$

$\eta$ - viscosidade da escória fundida;

$\theta$ - fração de fases sólidas precipitadas;

$\rho$ - parâmetro de interação do sólido (em suspensão diluída de esferas com diferentes tamanhos, o parâmetro é igual à unidade) [10].

Índice de espumação (Modelo de Skupien e Gaskell) [5] -

$\Sigma$ = Índice de espumação;

$\mu=$ viscosidade efetiva;

$\rho=$ densidade;

$\sigma=$ tensão superficial. 
Os valores de índice de espumação das escórias foram comparados com o THD de cada corrida de aço do FEA, para que fosse avaliada a influência da espumação no refino.

\section{RESULTADOS E DISCUSSÃO}

Para verificar a homogeneidade dos dados, foram plotadas as dispersões dos teores de $\mathrm{FeO}, \mathrm{MgO}$ e basicidade através de histogramas, para as 156 corridas conforme as Figura 5, Figura 6 e Figura 7, respectivamente.

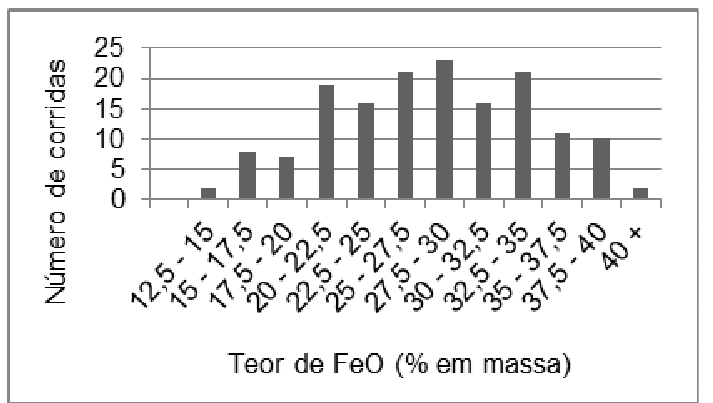

Figura 5. Distribuição do teor de FeO (\%) na escória do FEA.

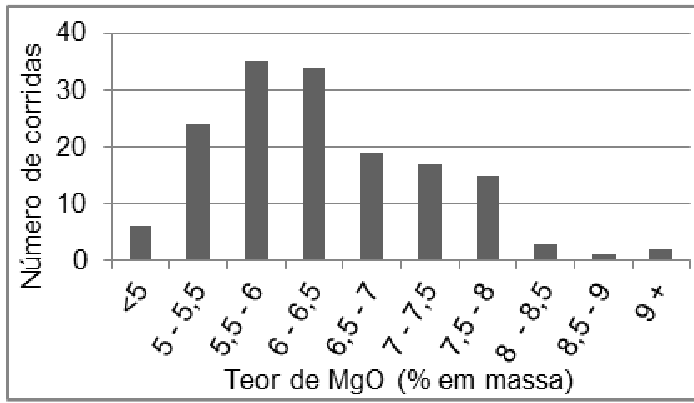

Figura 6. Distribuição do teor de MgO (\%) na escória do FEA.

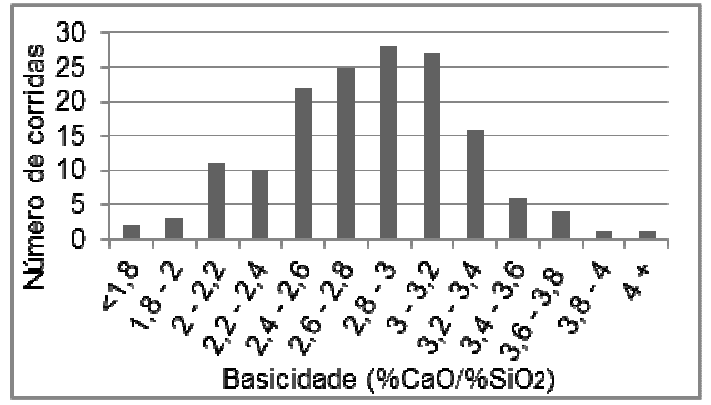

Figura 7. Distribuição da basicidade binária na escória do FEA.

Na Figura 5 observa-se aproximadamente $70 \%$ das corridas foram produzidas com o $\mathrm{FeO}$ entre 25 e 35\%. Estes teores correspondem às faixas ótimas para uma boa desfosforação e espumação da escória. Para o caso do MgO (figura 6) a faixa de composição química foi ainda mais estreita, ficando entre 5 e 6,5\%. Encontra-se na literatura que esta faixa pode representar a faixa de $\mathrm{MgO}$ necessária para a saturação de partículas sólidas, dependendo do teor de $\mathrm{FeO}$ e da basicidade [12]. A basicidade (figura 7) concentrou-se entre os valores de 2,4 e 3,2. Para que a espumação seja ideal, é preciso que a escória tenha um valor ótimo de basicidade, sendo que se esta for de baixa basicidade, será muito fluida, e se for extremamente básica, haverá um excesso de partículas sólidas, sendo então muito "dura", prejudicando a espumação da escória [12]. 
Os histogramas de "power off" e "power on" foram elaborados, para desconsiderar corridas que passaram por um tempo de parada muito longo, devido algum atraso de operação, afim de que não influenciassem nos resultados finais, Figura 8 e 9.

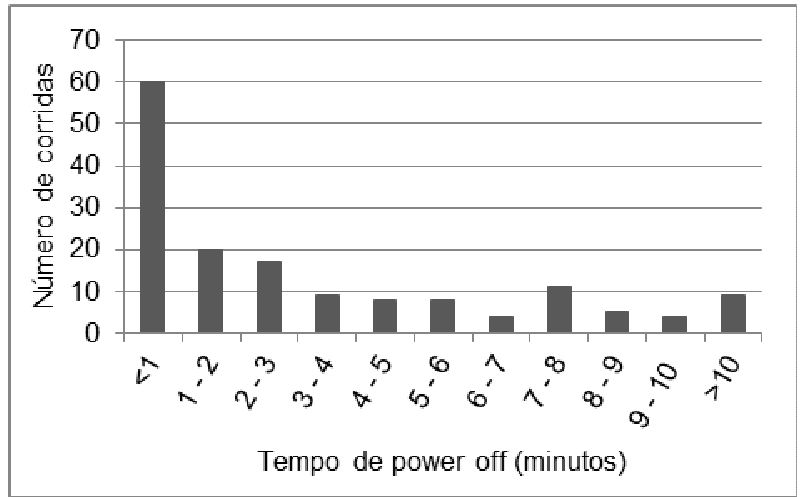

Figura 8. Distribuição do tempo de power off.

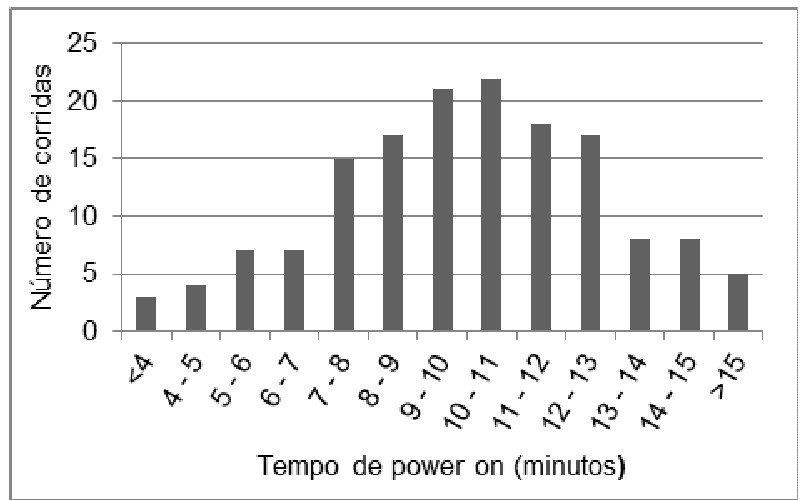

Figura 9. Distribuição do tempo de power on.

Na Figura 8,70\% das corridas tiveram um tempo de "power off" menores que 4 minutos. Já no caso do "power on", Figura 9, a maior parte das corridas está localizada em uma ampla faixa, com tempos de 7 a 13 minutos. "Power off" acima de 4 minutos e tempos de "power on" menores que 7 e maiores que 13 minutos foram desconsiderados, para uma melhor correlação.

A energia consumida no refino foi tabelada para as faixas que apresentaram maior concentração dos teores de $\mathrm{FeO}$ e basicidade, Tabelas 1 e 2 .

Tabela 1. Energias mínimas e máximas para as principais faixas de FeO.

\begin{tabular}{|ccc}
\hline Faixa de FeO (\%) & $\begin{array}{c}\text { Energia consumida } \\
\text { mínima no refino } \\
(\mathbf{K W h} / \mathbf{t})\end{array}$ & $\begin{array}{c}\text { Energia consumida } \\
\text { máxima no refino } \\
(\mathbf{K W h} / \mathbf{t})\end{array}$ \\
\hline $25,0-27,5$ & 42,46 & 108,14 \\
\hline $27,5-30,0$ & 42,14 & 87,51 \\
\hline $30,0-32,5$ & 62,79 & 114,09 \\
\hline $32,5-35,0$ & 66,91 & 103,26 \\
\hline
\end{tabular}

Pode-se dizer que os melhores resultados ocorreram para a faixa com valores entre 27,5 até $30 \%$ de $\mathrm{FeO}$, pois encontrou-se os menores pontos de máximo e mínimo para gasto energético. 
Tabela 2. Energias mínimas e máximas para as principais faixas de basicidade.

\begin{tabular}{ccc}
\hline $\begin{array}{c}\text { Faixa de } \\
\text { Basicidade } \\
\left(\% \mathrm{CaO} / \% \mathrm{SiO}_{2}\right)\end{array}$ & $\begin{array}{c}\text { Energia } \\
\text { consumida } \\
\text { mínima no } \\
\text { refino } \\
(\mathrm{KWh} / \mathrm{t})\end{array}$ & $\begin{array}{c}\text { Energia } \\
\text { consumida } \\
\text { máxima no } \\
\text { refino } \\
(\mathrm{KWh} / \mathrm{t})\end{array}$ \\
\hline $2,4-2,6$ & 71,75 & 108,54 \\
\hline $2,6-2,8$ & 69,02 & 97,59 \\
\hline $2,8-3,0$ & 67,68 & 114,09 \\
\hline $3,0-3,2$ & 51,74 & 94,24 \\
\hline
\end{tabular}

Pode-se observar que a faixa de basicidade que apresentou o melhor desempenho em termos de energia consumida durante o refino está entre 3 e 3,2, com gasto energético máximo de menor valor, de $94,24 \mathrm{KWh} / \mathrm{t}$, e o ponto de menor gasto energético, de 51,74 KWh/t. Conforme o estudo de Aminorroaya [8], foram encontradas faixas de $\mathrm{FeO}$ e basicidade ideais que promovem uma eficiente espumação onde se atinge um consumo energético mínimo.

Selecionando as corridas que apresentaram faixas de basicidades e teores de FeO ótimos para um menor consumo de energia elétrica, foi analisada a influência do teor de $\mathrm{MgO}$ no consumo de energia elétrica, Figura 10.

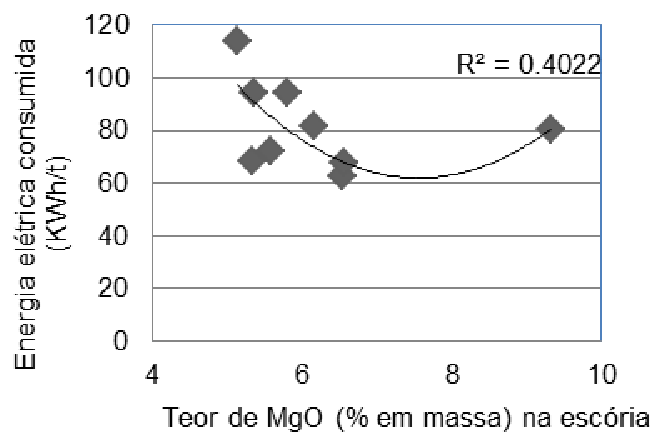

Figura 10. Teor de MgO e energia elétrica consumida.

Pode ser observar na figura 10 que o mínimo de energia consumida está próximo a $6,5 \% \mathrm{MgO}$.

O comportamento da tensão do arco elétrico e do THD foi avaliado durante a operação do FEA, um exemplo pode ser visto na Figura 11.
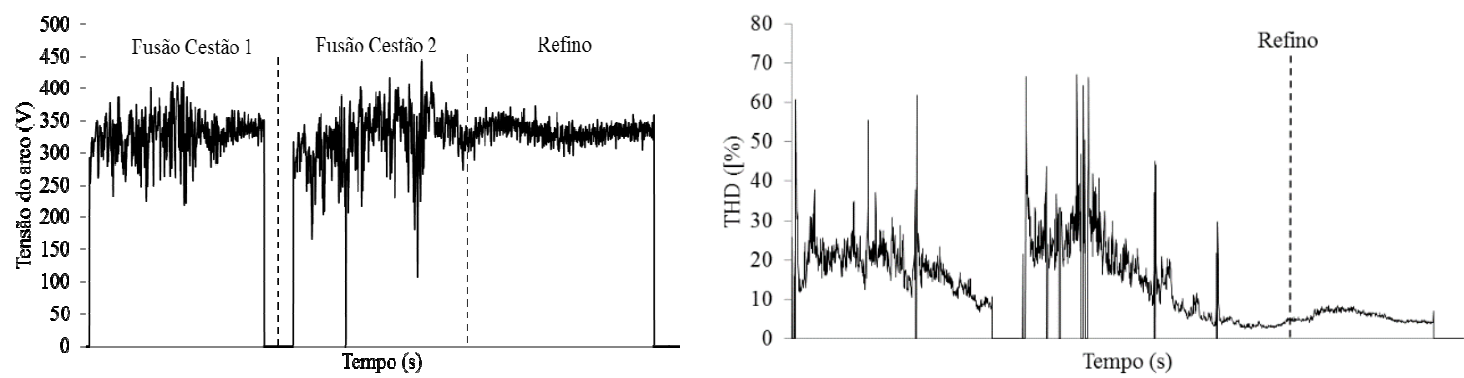

Figura 11. Comportamento da tensão do arco (esq.) e do THD (dir.) durante uma corrida.

A etapa de refino é indicada na Figura 11, mostrando que a estabilidade do arco aumenta com a presença de uma escória espumante, assim como Gandhare [1] descreve em seu estudo. As análises de desvio padrão da tensão pelo desvio padrão do THD foram feitas para as três fases do FEA, Figura 12. 
a)

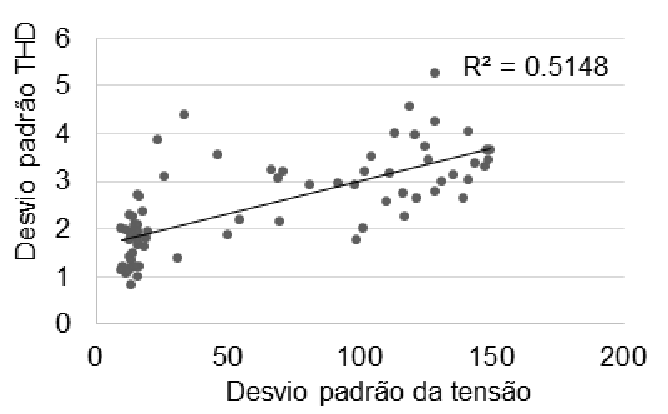

b)

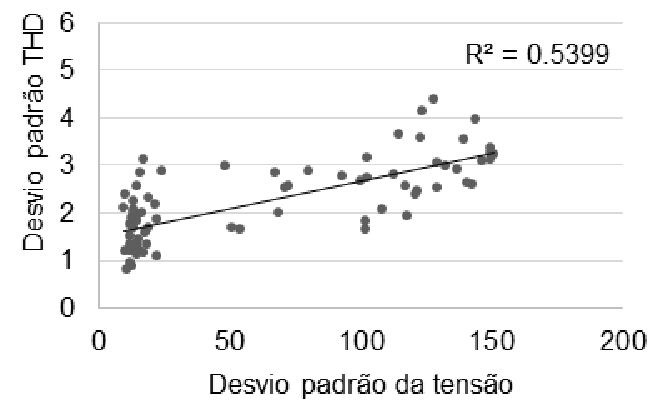

c)

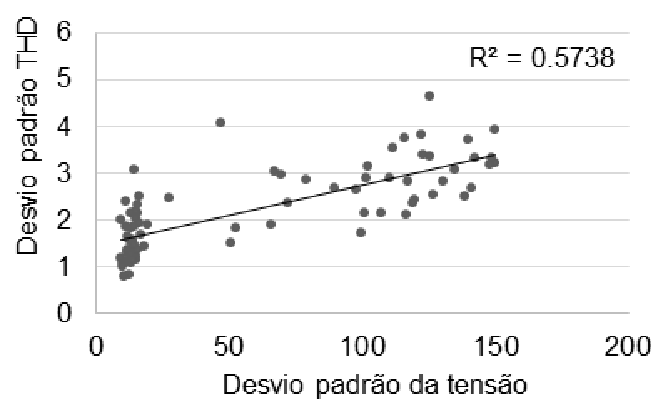

Figura 12. Relação entre o desvio padrão da tensão e o desvio padrão do THD para fase 1 (a), fase 2 (b) e fase 3 (c).

Nota-se a relação linear entre o desvio padrão da tensão e o desvio padrão do THD do FEA. Isso ocorre devido ao fato de quanto maior a instabilidade do arco elétrico, maior será o desvio da tensão e por consequência, maior a variação dos harmônicos de tensão. Foi feito então o gráfico do desvio padrão dos harmônicos pelo teor de $\mathrm{FeO}$, para as 3 fases, com o teor de $\mathrm{FeO}$ entre 25 e $35 \%$ e basicidade entre 2,4 e 3,2, Figura 13.

a)

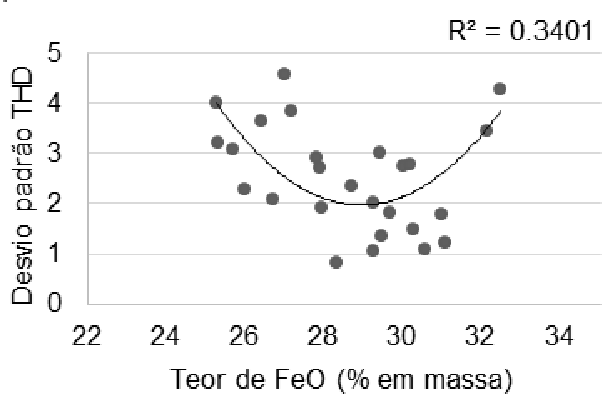

b)

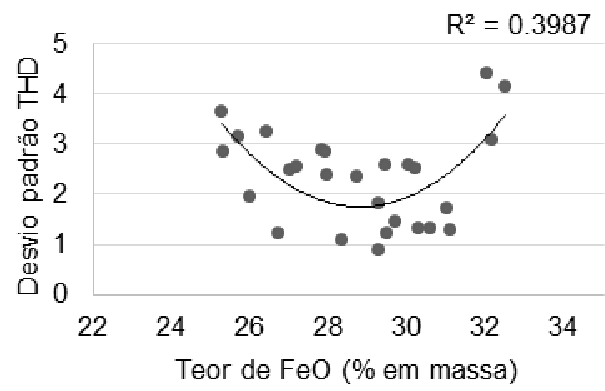

c)

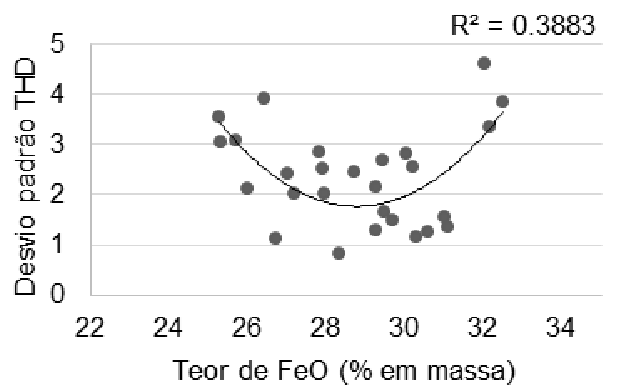

Figura 13. Relação entre o teor de FeO e o desvio padrão do THD fase 1 (a), fase 2 (b) e fase 3 (c). 
Foram selecionadas as corridas com teores de $\mathrm{FeO}$ entre $27,5-30$, basicidade entre 2,6 - 3,2, "power on" entre 7 e 13 minutos e "power off" abaixo de 4 minutos, pois apresentaram menor consumo de energia elétrica. As correlações entre 0 desvio do THD e a energia consumida podem ser vistas na Figura 14, para as três fases do FEA.

a)

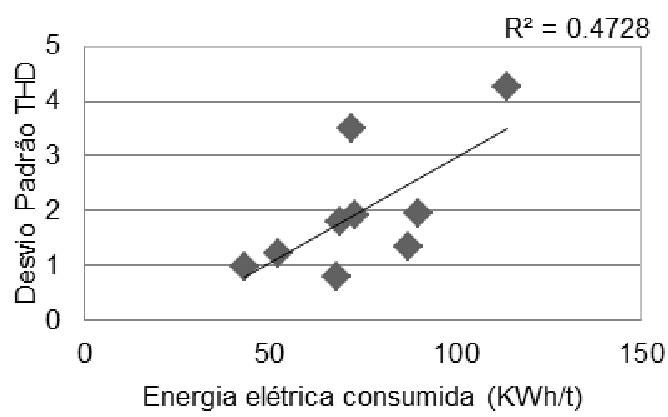

b)

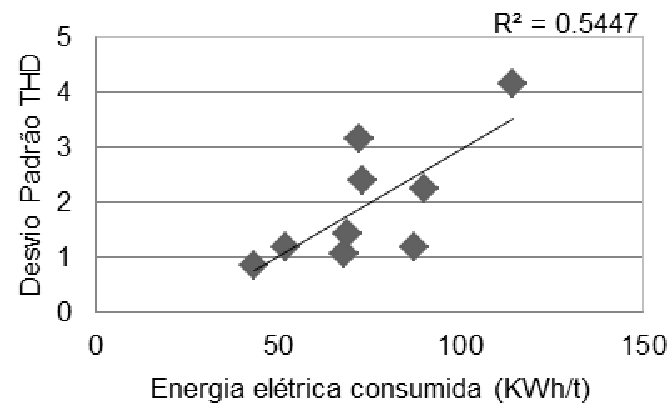

c)

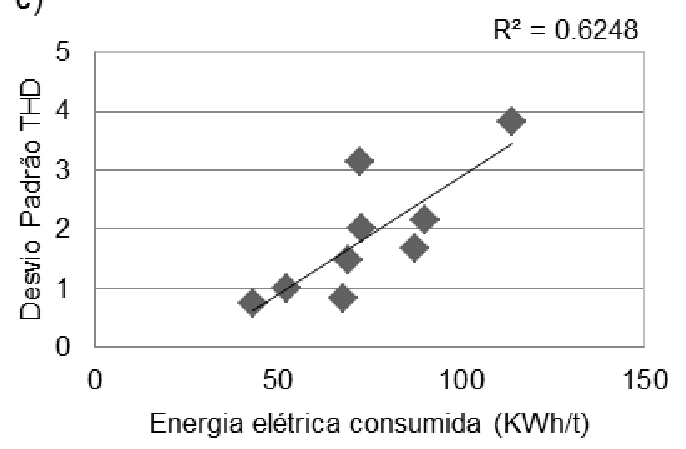

Figura 14. Energia elétrica consumida no refino e desvio padrão do THD para a fase 1 (a), fase 2 (b) e fase 3 (c).

Verifica-se que quanto menor o desvio padrão do THD, menor a distorção gerada pelos harmônicos, menor a instabilidade elétrica, e menor o consumo de energia elétrica.

A correlação entre o índice de espumação calculado e o desvio padrão da THD foi realizada, conforme Figura 15.

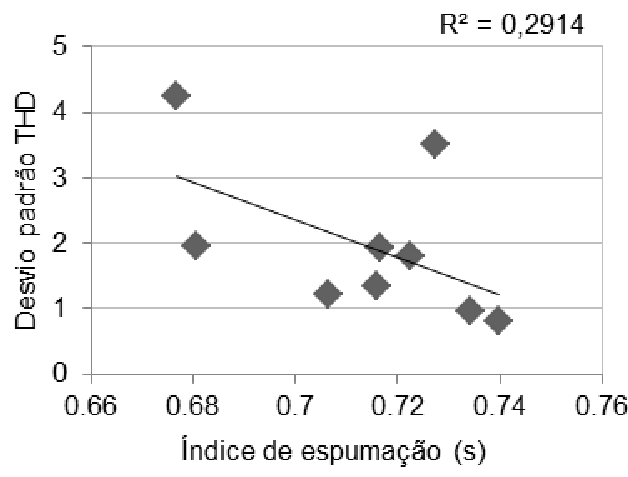

Figura 15. Índice de espumação calculado x Desvio padrão do THD.

Observa-se que a tendência é a esperada, onde ocorre a diminuição do THD com o aumento do índice de espumação calculado. 


\section{CONCLUSÕES}

Os valores de menor consumo de energia elétrica se encontraram para as faixas de:

- Teores de FeO entre 27,5 e 30\%;

- Basicidade entre 3,0 e 3,2;

- Teores de $\mathrm{MgO}$ entre 7 e $8 \%$, para as corridas que estão dentro da faixa ótima de FeO e basicidade.

$\mathrm{Na}$ análise dos dados elétricos:

- A relação entre o desvio padrão da tensão e do THD do arco se comportou de forma linear;

- O teor de FeO para um menor consumo de energia elétrica e menor THD foi de $29 \%$;

- Quanto maior o THD maior o consumo de energia elétrica no refino;

- A relação entre o índice de espumação e o THD apresentou tendência linear.

\section{Agradecimentos}

Deisi Vieira agradece ao professor Wagner Bielefeldt, pela orientação e apoio, ao professor Antônio Vilela pela oportunidade, à equipe do Laboratório de Siderurgia da UFRGS, à Fundação Luiz Englert e ao CNPq pelo suporte financeiro.

\section{REFERÊNCIAS}

1 Gandhare, W.Z. Analyzing Electric Power Quality in Arc Furnaces, International Conference on Renewable Energies and Power Quality, 2007.

2 Sedivy, C. Tools for Foaming Slag Operation at EAF Steelmaking. Issue 2, Vol 53, Austria, 2008.

3 Parsapoor, A. Predicting Foaming Slag Quality in Electric Arc Furnace Using Power Quality Indices and ANFIS. IEEE, Iran, 2010.

4 Ito K. Study on the Foaming of $\mathrm{CaO}_{-} \mathrm{SiO}_{2}-\mathrm{FeO}$ Slags. Vol 20 b, 1989.

5 Skupien, D. The Surface Tensions and Foaming Behavior of Melts in the System CaO$\mathrm{FeO}-\mathrm{SiO}_{2}$. Metallurgical and Materials Transactions, Vol 31B, 2000.

6 Bennett, J. Thermodynamic Studies of MgO Saturated EAF Slag. Ironmaking and Steelmaking, Vol 37, 2010.

7 Luz, A. P. Slag Foaming: Fundamentals, Experimental Evaluation and Application in the Steelmaking Industry. Refractories, World Forum, 2010.

8 Aminorroaya, S. The Effect of Foamy Slag in the Electric Arc Furnaces on Electric Energy Consumption. European Electric Steelmaking, 2002.

9 Mills, K.C, Keene, B.J. Physical Properties of BOS Slag. International Materials Reviews, Vol. 32, 1987.

10 R. Roscoe. The Viscosity of Suspensions of Rigid Spheres. Brit J Appl Phys, 1952, n. 3, pp. 267-269.

11 Paulino, M. A. S. et al. Obtenção de Escória Espumante Em Forno Elétrico a Arco: Determinação das Composições para o Sistema $\mathrm{CaO}-\mathrm{SiO}_{2}-\mathrm{MgO}-\mathrm{FeO}, 45^{\circ}$ Seminário de Aciaria - Internacional, Porto Alegre, Brasil, 2014.

12 Bielefeldt, W.V. Fundamentos de Escórias, 2012. 